\title{
A gastrointestinal stromal tumour presenting incidentally with haemorrhage and perforation associated with a Meckel's diverticulum: a case report
}

\author{
Richard Woolf $^{1 *}$, Natalie Blencowe ${ }^{1}$, Karim Muhammad ${ }^{1}$, David Paterson ${ }^{2}$ \\ and Geoff Pye P $^{3}$
}

\author{
Addresses: ${ }^{1}$ Department of Coloproctology, Bristol Royal Infirmary, Bristol, UK \\ ${ }^{2}$ Department of Histopathology, Weston General Hospital, Weston-super-Mare, UK \\ ${ }^{3}$ Department of Surgery, Weston General Hospital, Weston-super-Mare, UK \\ Email: RW* - woolfy28@hotmail.com; NB - natalieblencowe@doctors.org.uk; KM - kbmuhammad@hotmail.com; \\ DP - david.paterson@waht.swest.nhs.uk; GP - geoff.pye@waht.swest.nhs.uk \\ * Corresponding author
}

Received: 17 March 2008 Accepted: 2 February 2009 Published: 23 July 2009

Journal of Medical Case Reports 2009, 3:7423 doi: 10.4076/I752-1947-3-7423

This article is available from: http://jmedicalcasereports.com/jmedicalcasereports/article/view/7423

(c) 2009 Woolf et al.; licensee Cases Network Ltd.

This is an Open Access article distributed under the terms of the Creative Commons Attribution License (http://creativecommons.org/licenses/by/3.0), which permits unrestricted use, distribution, and reproduction in any medium, provided the original work is properly cited.

\begin{abstract}
Introduction: This is the first reported case of perforation and haemorrhage of a Meckel's diverticulum leading to the incidental finding of a gastrointestinal stromal tumour within the diverticulum. Meckel's diverticulum is the most common congenital abnormality of the gastrointestinal tract, however, when symptomatic, it is often misdiagnosed at presentation. Common complications presenting in adults include bleeding, obstruction, diverticulitis and perforation. Tumours within a Meckel's diverticulum are a rare but recognised complication. We discuss the management of a gastrointestinal tumour within the diverticulum.

Case presentation: A 59-year-old Caucasian man presented with acute right iliac fossa pain with localized peritonism. At surgery, he was found to have a perforated and haemorrhagic Meckel's diverticulum, associated with a gastrointestinal stromal tumour within the apex of the diverticulum. The absence of necrosis and a low mitotic rate indicated primary resection with subsequent computed tomography surveillance to be the most appropriate management strategy.

Conclusion: We report a unique triad of complications associated with the presentation of a Meckel's diverticulum. This article reviews this common congenital abnormality and discusses the management of a gastrointestinal tumour. Meckel's diverticulum will mimic other intra-abdominal pathologies in presentation and should therefore often be considered as a differential diagnosis.
\end{abstract}

\section{Introduction}

This is the first reported case of perforation and haemorrhage of a Meckel's diverticulum leading to the incidental finding of a gastrointestinal stromal tumour within the diverticulum. Meckel's diverticulum is the most common congenital abnormality of the gastrointestinal tract, however, when symptomatic, it is often misdiagnosed at presentation. Common complications presenting 
in adults include bleeding, obstruction, diverticulitis and perforation. Tumours within a Meckel's diverticulum are a rare but recognised complication.

\section{Case presentation}

A 59-year-old Caucasian man presented with peri-umbilical pain that had localized to the right iliac fossa. On examination, he was tender in the right iliac fossa, with localized peritonism. His white cell count was $10.2 \times 10^{9}$ (neutrophils $8.1 \times 10^{9}$ ) and with C-reactive protein (CRP) $<5$. Acute appendicitis was diagnosed clinically and a diagnostic laparoscopy performed.

A perforated Meckel's diverticulum was found, associated with free intra-abdominal fluid and haemorrhage. At subsequent laparotomy, $75 \mathrm{~mm}$ of small bowel was resected and primary anastamosis was performed. Histology confirmed a Meckel's diverticulum and with a $25 \mathrm{~mm}$ area of perforation (Figures 1-3). An incidental finding was a $45 \mathrm{~mm}$ nodule at the apex of the diverticulum with the following features:

1. Full thickness tumour of the bowel wall, extending to serosal surfaces (Figure 4).

2. No areas of tumour necrosis.

3. Less than one mitotic figure in $10 \times 40$ high powered fields.

4. Interlacing bundles of spindle cells with elongated blunt ended nuclei.

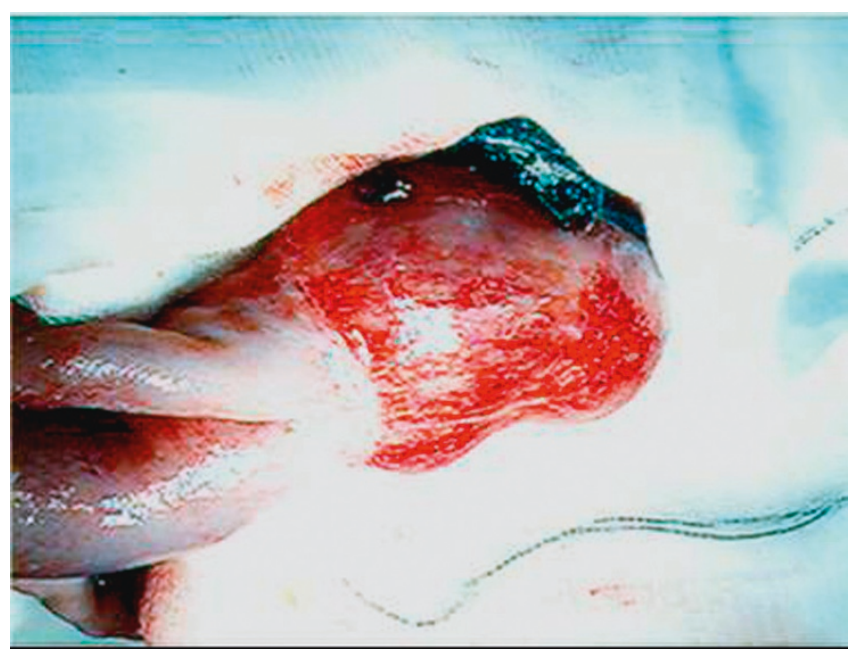

Figure I. Intra-operative photograph demonstrating Meckel's diverticulum and overlying thrombus.

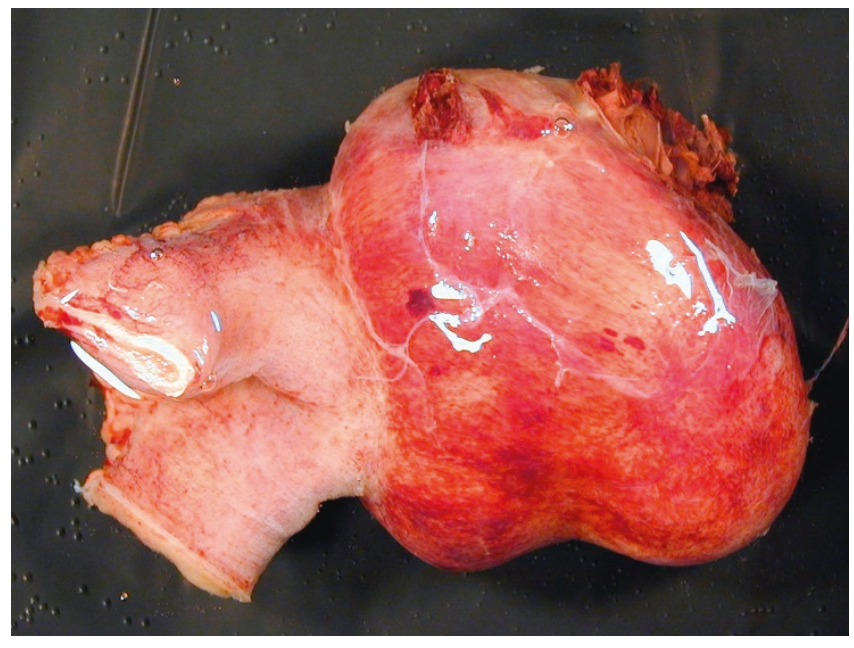

Figure 2. Resected specimen.

5. Some nuclear variability and tumour giant cells present.

6. Positive for CD117 and smooth muscle actin (Figures 5 and 6), negative for S100 protein and cytokeratin.

This was, therefore, confirmed to be a GIST. Resection margins were found to be complete. The patient received 72 hours of intravenous antibiotics and made a good recovery. Surveillance abdominal computed tomography (CT) scan at one year was unremarkable.

\section{Discussion}

Meckel's diverticulum is a congenital abnormality that arises at the site of the vitelline duct, which in the embryo

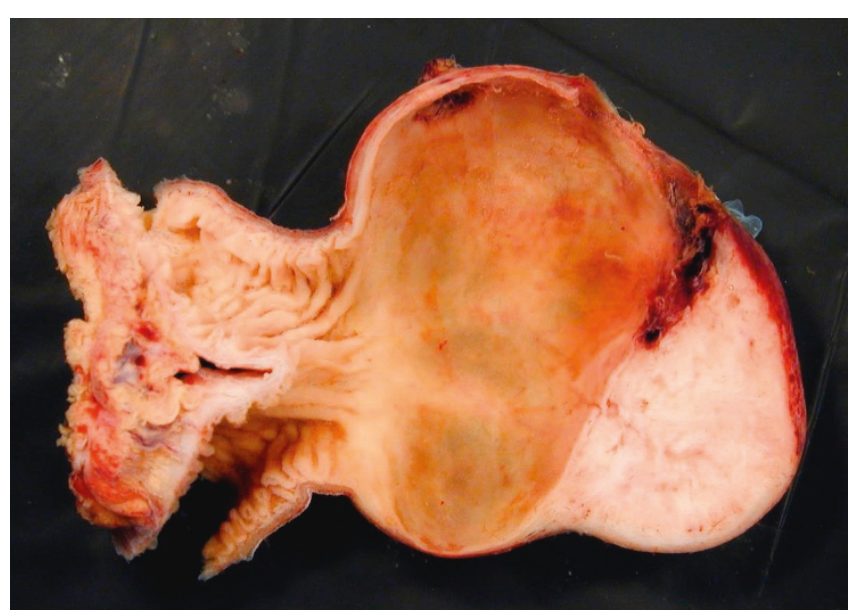

Figure 3. Resected specimen in cross-section demonstrating the perforated wall of the Meckel's diverticulum at the superior margin and the gastrointestinal stromal tumour at the apex of the diverticulum. 


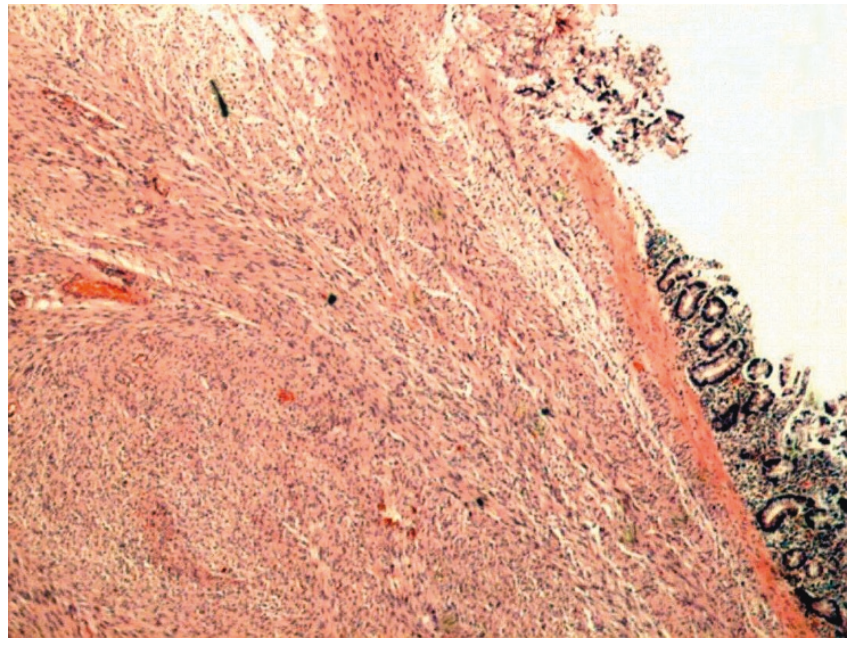

Figure 4. Light micrograph of histological specimen demonstrating full thickness tumour of the bowel wall extending to the serosal surface (haematoxylin and eosin stain; $\times 100$ ).

connects the primitive gut to the yolk sac. If this fails to obliterate by the seventh week of gestation, congenital defects can persist that include umbilical sinus, omphalomesenteric fistula, enterocyst, fibrous band and most commonly Meckel's diverticulum. The diverticulum is a true diverticulum containing all layers of the intestinal wall and most commonly arises from the antimesenteric aspect of the ileum, proximal to the ileocaecal valve. It has an independent blood supply from a remnant of the vitelline artery, a branch of the superior mesenteric artery.

The diverticulum is commonly described by the rule of ' $2 s^{\prime}$ : occurring in $2 \%$ of the population, approximately

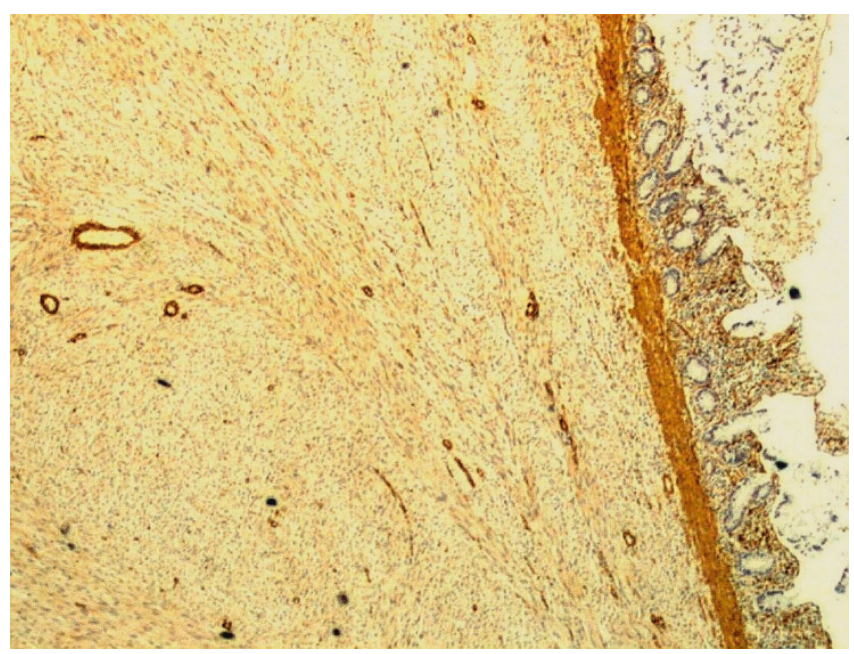

Figure 5. Light micrograph with smooth muscle actin staining showing uptake by tumour tissue $(\times 100)$.

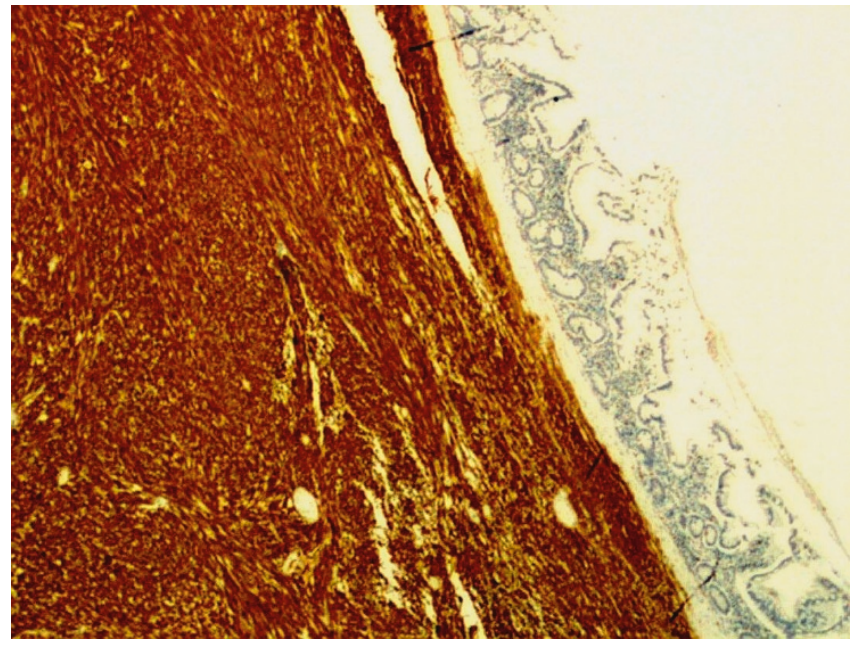

Figure 6. Light micrograph with CDII7 staining showing uptake by tumour tissue $(\times 100)$.

2 inches long, arising within 2 feet of the ileocaecal valve, commonly affecting children less than 2 years of age and occurring twice as often in men [1]. Although approximate guidelines describe this anatomical variant, they are not accurate. The diagnosis of a Meckel's diverticulum is often incidental at laparotomy or laparoscopy and its prevalence is only approximated at $2 \%$. Some autopsy studies suggest that the true percentage may be higher at $4.5 \%$ [1]. Ninety percent of the diverticula are less than $10 \mathrm{~cm}$ long but cases have been reported of up to $100 \mathrm{~cm}$ [2]. Meckel's diverticula occur in the terminal ileum but their distance from the ileocaecal valve has been found to increase with age with an average distance of $34 \mathrm{~cm}$ in children under the age of 2 years, but $64 \mathrm{~cm}$ in adults [3].

Meckel's diverticulum can either be an incidental finding or present symptomatically. It is thought that between $4.2 \%$ and $6.4 \%$ become symptomatic, with the incidence falling with increasing age [4-6]. The mean age of presentation of symptomatic Meckel's diverticulum is 31 years with a male to female ratio of 3:1 (in both adult and paediatric groups), however, the incidental diagnosis has a more equal sex distribution in adults [6]. In a large series of 1476 cases at the Mayo Clinic, Park et al. report the most common presentations of symptomatic Meckel's diverticula in adults to be bleeding (38\%), obstruction (34\%), diverticulitis (28\%) and perforation (10\%) [6]. Perforation is most common secondary to ulceration of ectopic gastric mucosa, foreign bodies or Littre's hernia [7]. Due to the rarity of this anatomical abnormality, symptomatic Meckel's diverticula are misdiagnosed in approximately $90 \%$ of cases and acute appendicitis is the usual preoperative diagnosis [8]. 
Tumours are reported to occur in $0.5 \%$ and $3.2 \%$ of symptomatic Meckel's diverticula [8]. In a review of reported cases since 1965, Hager et al. state the prevalences of tumours of Meckel's diverticula to be: carcinoid tumour $(31.5 \%)$, leiomyosarcoma $(25.5 \%)$, adenocarcinoma (11.4\%) and leiomyoma (9.4\%) with overall malignancy in $77 \%$ of cases [7]. The definition of a gastrointestinal stromal tumour (GIST) has varied since the first use of the term in 1983. Originally, it encompassed gastrointestinal non-epithelial neoplasms lacking the immunohistochemical features of Schwann cells and did not have the ultrastructural characteristics of smooth muscle cells [9]. Using this original classification of GIST, $42 \%$ of all tumours and $41 \%$ of malignant tumours of Meckel's diverticula would be classified as GIST [7]. However, GIST is now recognised as a separate tumour entity and is defined as a spindle cell, epithelioid or pleiomorphic mesenchymal tumour of the gastrointestinal tract that strongly expresses the KIT (CD 117) protein and may harbour mutations of the type III tyrosine kinase receptor gene (either KIT or PDGFRA) [10].

GIST accounts for $0.1 \%$ and $3 \%$ of all gastrointestinal neoplasms, most commonly occurring in the stomach or small bowel, and is now the most common sarcoma of the small intestine [10]. Small bowel GISTs have a range of presenting features, including abdominal pain, an abdominal mass, gastrointestinal bleeding, small bowel obstruction, weight loss, fever, abscess or perforation [7].

There are little prognostic data regarding GISTs and current prognostic indicators are based on consensus guidelines. The most important adverse factors are thought to be a tumour diameter of greater than $5 \mathrm{~cm}$ and a high mitotic count exceeding five mitotic figures per 50 high powered fields on light microscopy $[10,11]$. Other suggested factors indicative of poor prognosis include tumour perforation, tumour necrosis, high cellularity and marked pleiomorphism [10]. The case reported by us has a low risk of recurrence based on a maximum diameter of $4.5 \mathrm{~cm}$, a low mitotic count of less than one mitotic figure in $10 \times 40$ high powered fields, and no evidence of necrosis. Importantly, the perforation of the diverticulum was also not associated with the tumour nodule. Surgery is considered the standard treatment for non-metastatic GIST with enbloc resection and clear margins. Study data on GISTs presenting in the United States between 1992 and 2000 state a 5-year survival of 50-60\% after complete resection of the localized primary tumour [12]. There is little evidence supporting local/regional lymphadenectomy as GISTs rarely metastasize to lymph nodes [10]. Targeted therapy with Imantinib, a KIT tyrosine kinase inhibitor, is considered the standard treatment for metastatic GIST [10].

\section{Conclusion}

Since 1978, there have been approximately 10 reported cases of tumour-associated perforation of a Meckel's diverticulum, however, only two of these were histologically classified as GISTs $[7,13]$. Our case is the first reported patient with perforation of a Meckel's diverticulum with frank intra-abdominal haemorrhage that led to the incidental discovery of the separate pathology of GIST within the diverticulum. Meckel's diverticulum can mimic other intra-abdominal pathologies in presentation and should therefore be considered as a differential diagnosis.

\section{Abbreviations}

CRP, C-reactive protein; CT, computed tomography; GIST, gastrointestinal stromal tumour.

\section{Consent}

Written informed consent was obtained from the patient for publication of this case report and any accompanying images. A copy of the written consent is available for review by the Editor-in-Chief of this journal.

\section{Competing interests}

The authors declare that they have no competing interests.

\section{Authors' contributions}

RW conceived the original article design, drafted the original manuscript reviewing current literature and corresponded with the editorial team. NB summarized the case and helped substantially with the drafting and critiquing of the original manuscript. KB and GP were the surgeons who operated on the patient, and helped substantially with critiquing of the original manuscript. DP examined the histological specimens and provided the histological diagnosis. All authors read and approved the final manuscript.

\section{Acknowledgements}

The authors would like to thank the patient for allowing us to publish this report.

\section{References}

I. Dumper J, Mackenzie S, Mitchell P, Sutherland F, Quan ML, Mew D: Complications of Meckel's diverticula in adults. Can J Surg 2006, 49:353-357.

2. Moses WR: Meckel's diverticulum. Report of two unusual cases. N Engl J Med 1947, 237: I I8-I22.

3. Yamaguch M, Takeuchi S, Awazu S: Meckel's diverticulum. Investigation of 600 patients in Japanese literature. Am J Surg 1978, 136:247-249.

4. Soltero MJ, Bill AH: The natural history of Meckel's Diverticulum and its relation to incidental removal A study of 202 cases of diseased Meckel's Diverticulum found in King County, Washington, over a fifteen year period. Am J Surg 1976, I32:168-I73.

5. Cullen JJ, Kelly KA, Moir CR, Hodge DO, Zinsmeister AR, Melton LJ 3rd: Surgical management of Meckel's diverticulum. An epidemiologic, population-based study. Ann Surg 1994, 220:564-568. 
6. Park J, Wolff B, Tollefson M, Walsh E, Larson D: Meckel diverticulum. The Mayo Clinic experience with 1476 patients (1950-2002). Ann Surg 2005, 24I:529-533.

7. Hager M, Maier $H$, Eberwein M, Klingler P, Kolbitsch C, Tiefenthaler W, Mikuz G, Moser PL: Perforated Meckel's diverticulum presenting as a gastrointestinal stromal tumor: a case report. J Gastrointest Surg 2005, 9:809-8I I.

8. Yahchouchy E, Marano A, Etienne J, Fingerhurt A: Meckel's diverticulum. I Am Coll Surg 200I, 192:658-662.

9. Mazur M, Clark H: Gastric stromal tumors: Reappraisal of histiogenesis. Am J Surg Pathol 1983, 7:507-5।9.

10. Joensuu H: Gastrointestinal stromal tumour (GIST). Ann Oncol 2006, 10:280-286.

II. Chang M, Choe G, Kim W, Kim Y: Small intestine stromal tumours: a clinicopathological study of 3 I tumours. Pathol Int 1998, 48:34I-347.

12. Tran T, Davila J, El-Serag H: The epidemiology of malignant gastrointestinal tumours: an analysis of I458 cases from I992 to 2000. Am J Gastroenterol 2005, 100:162-168.

13. Szentpali K, Palotas A, Wolfard A, Tiszlavicz L, Balogh A: A gastrointestinal stromal tumour presenting in a perforated Meckel's diverticulum. Can J Surg 2004, 47:70.

\section{Do you have a case to share?}

Submit your case report today

- Rapid peer review

- Fast publication

- PubMed indexing

- Inclusion in Cases Database

Any patient, any case, can teach us something

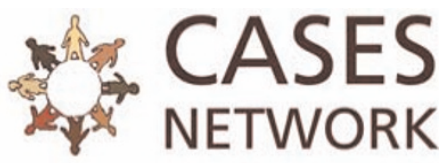

www.casesnetwork.com 